\title{
Contact Potentials, Fermi Level Equilibration, and Surface Charging
}

\author{
Pekka Peljo, ${ }^{*} \dagger$ José A. Manzanares, ${ }^{\ddagger}$ and Hubert H. Girault ${ }^{\dagger}$ \\ ${ }^{\dagger}$ Laboratoire d’Electrochimie Physique et Analytique, École Polytechnique Fédérale de Lausanne, EPFL Valais Wallis, Rue de \\ l'Industrie 17, Case Postale 440, CH-1951 Sion, Switzerland \\ ${ }^{\ddagger}$ Department of Thermodynamics, Faculty of Physics, University of Valencia, c/Dr. Moliner, 50, E-46100 Burjasot, Spain
}

\section{Supporting Information}

ABSTRACT: This article focuses on contact electrification from thermodynamic equilibration of the electrochemical potential of the electrons of two conductors upon contact. The contact potential difference generated in bimetallic macroand nanosystems, the Fermi level after the contact, and the amount and location of the charge transferred from one metal to the other are discussed. The three geometries considered are spheres in contact, Janus particles, and core-shell particles. In addition, the force between the two spheres in contact with each other is calculated and is found to be attractive. A simple electrostatic model for calculating charge distribution and potential profiles in both vacuum and an aqueous electrolyte solution is described. Immersion of these bimetallic systems into an electrolyte solution leads to the formation of an electric double layer at the metal-electrolyte interface. This Fermi level equilibration and the associated charge transfer can at least partly explain experimentally observed different electrocatalytic, catalytic, and optical properties of multimetallic nanosystems in comparison to systems composed of pure metals. For example, the shifts in the surface plasmon resonance peaks in bimetallic core-shell particles seem to result at least partly from contact charging.

\section{INTRODUCTION}

Although observation of potential differences when two metals are placed in contact dates back from the late 18th century "electricians" and of course to the seminal work of Alessandro Volta, the actual effects of the contact electrification are often neglected. Although bimetallic nanoparticles have recently received increasing attention because of their promising electrocatalytic, ${ }^{1-3}$ catalytic, ${ }^{4-8}$ and optical properties, ${ }^{4,9}$ it is equally important to understand how contact electrification can change and contribute to enhance these properties. The interaction of nanoparticles with their environment can, sometimes drastically, shift the Fermi level of electrons in the nanoparticles and influence their chemical and electrochemical properties as highlighted in a review by Scanlon et al. ${ }^{10}$ Contact electrification can also significantly shift the Fermi level of electrons in bi- or multimetallic structures in comparison with pure materials. This is important because these shifts in the Fermi level can have a drastic effect on the properties of multimetallic systems. For example, shifts observed in surface plasmon resonance of core-shell nanoparticles seem to result from contact electrification, as described vide infra.

The aim of this article is to clarify the position of the Fermi level after contact charging, to understand how much charge is transferred and where this charge is located, to give some idea about how these effects influence properties of these systems, and to illustrate what happens when these particles are immersed in an electrolyte solution, leading to the formation of an electric double layer around the particles. In addition, the forces between two spheres in contact are described.

To understand what happens upon metal-metal contact and how this phenomenon affects, for example, electrocatalytic, catalytic, and optical properties of bimetallic nanosystems, we must understand the governing principles. However, electrochemists use different terms than solid state and semiconductor physicists, giving rise to additional confusion. In this article, we describe the thermodynamics of the contact electrification from the point of view of an electrochemist.

The history of the contact electrification until year 1900 has been comprehensively reviewed by Hong. ${ }^{11}$ The accurate measurement of the contact potential difference dates back to the works of Hankel, Pellat, and Kelvin. ${ }^{12}$ Hankel studied an apparatus based on a capacitor with two plates of different materials, charging itself upon contact. When one plate was grounded and the other was insulated, the potential difference increased with an increase in the separation distance between plates, enabling the measurement of the contact potential. Pellat improved this method by connecting the first plate to a potentiometer to allow compensation of the contact potential. This so-called null method is very accurate, because even small, uncompensated potentials resulted in measurable forces between the capacitor plates, and this method was named

Received: April 4, 2016

Revised: $\quad$ May 9, 2016

Published: May 13, 2016 
after Kelvin. The sensitivity of the Kelvin probe was further increased by Zisman ${ }^{13}$ using vibrating plates coupled with headphones as the detecting instrument. The state-of-the-art system is now the Kelvin probe force microscope, allowing the measurement of the local contact potential difference between a conducting atom force microscopy tip and the sample. This technique allows the mapping of the surface topography and local work function with high spatial resolution. For further details, the reader is directed to the review by Lee et al. ${ }^{14}$

The triboelectric electrostatic charging by friction has also been covered in a review by Lacks and Sankaran, ${ }^{15}$ and is out of scope of this article focusing on the thermodynamics of the contact electrification. In 1951, Harper ${ }^{16}$ studied contact electrification of different metal spheres. He demonstrated that the amount of charge transferred upon contact of two different metal spheres showed almost a linear relationship with the contact potential difference between the two metals. To eliminate the effects of the triboelectric charging, the contact was achieved by a floating sphere apparatus, and the VoltaHelmholtz hypothesis that "the charge obtained on separating two bodies must have been present as a double layer when they were in contact" was corroborated. ${ }^{16}$ These results clearly show that when the separation between two spheres is increased, the capacitance of the system changes: first, the charge transfer occurs to keep the Fermi levels of both spheres equal, and then at a certain threshold value, the tunneling of electrons is no longer possible between the two spheres, and the charge remains constant. ${ }^{16}$ Because the capacitance of the system varies as a function of the separation, the electrostatic interactions between the two equal spheres redistribute the surface charge, so that they can effectively be considered as large dipoles. ${ }^{17}$ These large dipoles attract each other unless they have exactly the same charge (in this case they repel each other). ${ }^{17}$

This article focuses on the contact electrification from thermodynamic equilibration of the electrochemical potential of the electrons of two conductors upon contact. We have recently proposed that the contact electrification in bimetallic segregated systems can be quantitatively understood by simply considering the system as a nanocapacitor, where a thin vacuum layer separates the two metals. The potential difference between the two metals is directly given by the work function difference, and the amount of transferred charge can be calculated simply from electrostatics. ${ }^{18}$ Comparison between numerical simulations of the electrostatics and more comprehensive density functional theory (DFT) calculations showed that the continuum calculations can satisfactorily describe the general charge transfer behavior, but DFT calculations are required to accurately describe the atomic scale charge transfer at the metal-vacuum interface and to resolve the finer details of transferred charge at the metalmetal interface. ${ }^{18}$

\section{ELECTROSTATICS OF CONDUCTORS WITH DIFFERENT WORK FUNCTIONS}

Fermi Level Equilibration of Metallic Spheres. According to its IUPAC definition, ${ }^{19-23}$ the electrochemical potential $\tilde{\mu}_{\mathrm{e}^{-}}$in a conducting phase is the work of bringing one electron from a reference point in vacuum (out of the range of the charge of the phase) to the bulk phase; herein, we consider chemical potentials per particle, not molar values. The electrochemical convention assigns the zero energy to an electron at rest in a field-free vacuum. Then, the electronic energies in a metal have negative values. The work to bring this electron from the reference point to just outside of the metal is $-e \psi{ }^{20}$ The work function $\Phi=-e \psi-\tilde{\mu}_{\mathrm{e}^{-}}$is the difference in the energies of the electron just outside of the metal $(-e \psi)$ and inside of the metal $\left(\tilde{\mu}_{\mathrm{e}^{-}}\right)$. The ionization energy (IE) is defined as the work to remove one electron from a particle. When the state of charge is not significantly affected by the removal of an electron, the IE can be approximated by

$$
\mathrm{IE}=-\tilde{\mu}_{\mathrm{e}^{-}}=\Phi+e \psi
$$

The electrostatic term $e \psi$ indicates that it is more difficult to extract electrons from a particle that is positively charged. The basic concepts are further reviewed in the Supporting Information.

Electrons tend to flow in the direction that decreases the gradient of their electrochemical potential until it vanishes. This process is known as Fermi level equilibration. In the case of two conductors $\mathrm{A}$ and $\mathrm{B}$, the equilibrium condition is $\tilde{\mu}_{\mathrm{e}^{-}}^{\mathrm{A}}=\tilde{\mu}_{\mathrm{e}^{--}}^{\mathrm{B}}{ }^{20}$ This equilibrium is achieved through charge redistribution, and hence it involves the electrostatic energy of the electrons. Thus, the equilibrated Fermi level should be understood as the electrochemical potential of the electrons, $E_{\mathrm{F}}=\tilde{\mu}_{\mathrm{e}^{-} ;}{ }^{21,24}$ this is also the highest occupied energy level at $0 \mathrm{~K}$ provided that these levels include the electrostatic potential energy.

When two conductors of different metals A and B carry charges $q^{\mathrm{A}}$ and $q^{\mathrm{B}}$, their energy is

$$
W=\frac{1}{e}\left(q^{\mathrm{A}} \Phi^{\mathrm{A}}+q^{\mathrm{B}} \Phi^{\mathrm{B}}\right)+\frac{1}{2}\left(q^{\mathrm{A}} \psi^{\mathrm{A}}+q^{\mathrm{B}} \psi^{\mathrm{B}}\right)
$$

In the capacitance matrix formalism, the charges on the conductors are expressed in terms of their (outer) electrostatic potentials and the self- and mutual capacitances as ${ }^{17}$

$$
\left(\begin{array}{l}
q^{\mathrm{A}} \\
q^{\mathrm{B}}
\end{array}\right)=\left(\begin{array}{ll}
C_{\mathrm{AA}} & C_{\mathrm{AB}} \\
C_{\mathrm{AB}} & C_{\mathrm{BB}}
\end{array}\right)\left(\begin{array}{l}
\psi^{\mathrm{A}} \\
\psi^{\mathrm{B}}
\end{array}\right)
$$

At constant total charge $q_{\mathrm{T}} \equiv q^{\mathrm{A}}+q^{\mathrm{B}}$ and fixed spatial configuration of the conductors, the equilibrium condition $\left(\partial W / \partial q^{\mathrm{A}}\right)_{q_{\mathrm{T}}, C_{i j}}=0$ with respect to the exchange of charge is achieved when the Volta potential difference is

$$
\Delta_{\mathrm{A}}^{\mathrm{B}} \psi_{\mathrm{eq}} \equiv\left(\psi^{\mathrm{B}}-\psi^{\mathrm{A}}\right)_{\mathrm{eq}}=-\left(\Phi^{\mathrm{B}}-\Phi^{\mathrm{A}}\right) / e \equiv-\Delta_{\mathrm{A}}^{\mathrm{B}} \Phi / e
$$

From eq 1 , this is equivalent to the Fermi level equilibration $\tilde{\mu}_{\mathrm{e}^{-}}^{\mathrm{A}}=\tilde{\mu}_{\mathrm{e}^{-}}^{\mathrm{B}}$. Thus, the outer potentials of two conductors in equilibrium differ in a quantity proportional to the difference in their work functions. If the conductors were initially uncharged, electrons would flow from the conductor with the lowest work function to the other conductor until their Fermi levels equalize. $^{20}$

The total charge $q_{\mathrm{T}}$ determines the average potential $\psi_{\mathrm{av}} \equiv$ $c_{\mathrm{A}} \psi^{\mathrm{A}}+c_{\mathrm{B}} \psi^{\mathrm{B}}=q_{\mathrm{T}} / C_{\mathrm{T}}$ of the conductors, where $C_{\mathrm{T}} \equiv C_{\mathrm{AA}}+$ $2 C_{\mathrm{AB}}+C_{\mathrm{BB}}$ and $c_{\mathrm{A}} \equiv\left(C_{\mathrm{AA}}+C_{\mathrm{AB}}\right) / C_{\mathrm{T}} \equiv 1-c_{\mathrm{B}}$. When the conductors have opposite charges $q^{\mathrm{B}}=-q^{\mathrm{A}} \equiv q$, the average potential vanishes and their potential difference is given by $q=$ $C \Delta_{\mathrm{A}}^{\mathrm{B}} \psi$, where

$$
C \equiv \frac{C_{\mathrm{AA}} C_{\mathrm{BB}}-\left(C_{\mathrm{AB}}\right)^{2}}{C_{\mathrm{AA}}+2 C_{\mathrm{AB}}+C_{\mathrm{BB}}}
$$



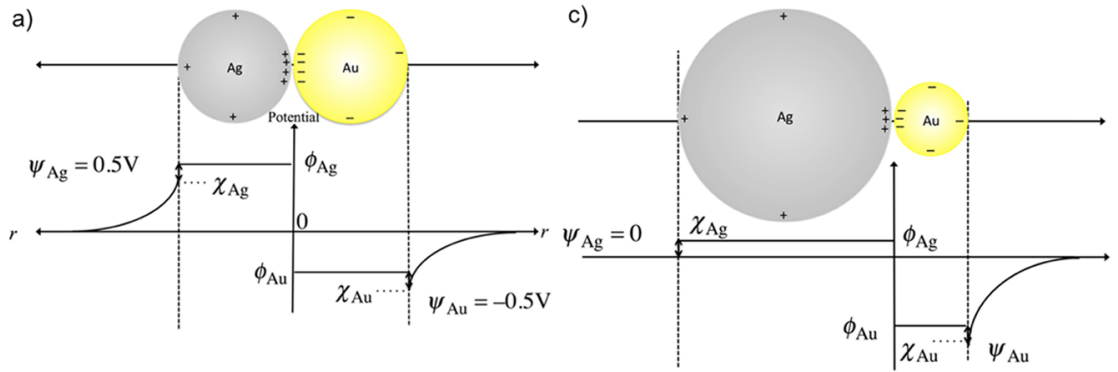

b)

Electron at rest in vacuum

d)
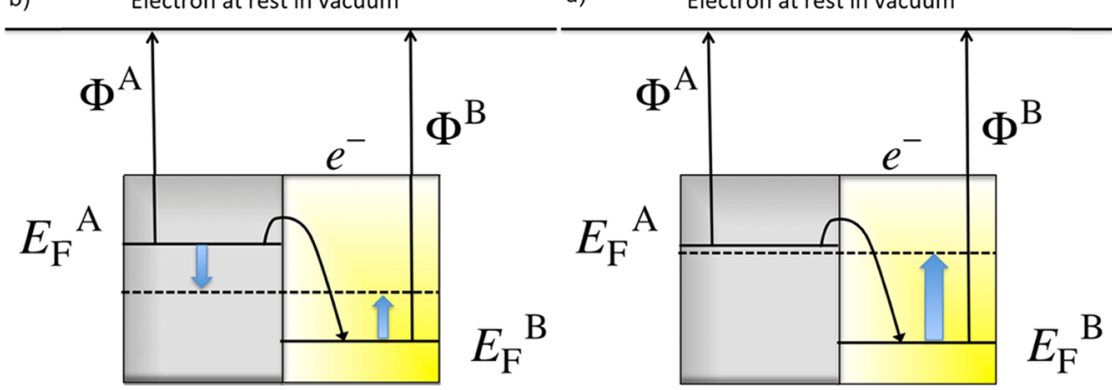

Figure 1. (a) Potential distribution along the symmetry axis through the contact point of two spheres of Ag and Au. The inner or Galvani potential of a phase is the sum of the surface potential and the outer potential, $\phi=\chi+\psi$. The surface potential $\chi^{\mathrm{Ag}}$ is not necessarily equal to $\chi^{\mathrm{Au}}$. (b) Work functions of the metals $(\mathrm{A}=\mathrm{Ag}, \mathrm{B}=\mathrm{Au})$ and illustration of the Fermi level equilibration by electron flow upon contact. (c) Potential distribution for a small gold sphere and a large silver sphere. (d) Equilibration of Fermi levels upon contact corresponding to panel $c$.

is known as the system capacitance. ${ }^{17,25}$ In general, the Volta potential difference is given by $C \Delta_{\mathrm{A}}^{\mathrm{B}} \psi=c_{\mathrm{A}} q^{\mathrm{B}}-c_{\mathrm{B}} q^{\mathrm{A}}$, so that the condition $\psi^{\mathrm{A}}=\psi^{\mathrm{B}}$ is equivalent to $c_{\mathrm{A}} q^{\mathrm{B}}=c_{\mathrm{B}} q^{\mathrm{A}}$. ${ }^{17}$ The latter general expression for $\Delta_{\mathrm{A}}^{\mathrm{B}} \psi$ is equivalent to $q^{\mathrm{A}}=c_{\mathrm{A}} q_{\mathrm{T}}-C \Delta_{\mathrm{A}}^{\mathrm{B}} \psi$ and $q^{\mathrm{B}}=q_{\mathrm{T}}-q^{\mathrm{A}}$. With these expressions, the system energy can also be represented as

$$
\begin{aligned}
W= & C \Delta_{\mathrm{A}}^{\mathrm{B}} \psi\left(\frac{1}{e} \Delta_{\mathrm{A}}^{\mathrm{B}} \Phi+\frac{1}{2} \Delta_{\mathrm{A}}^{\mathrm{B}} \psi\right)+\frac{\left(q_{\mathrm{T}}\right)^{2}}{2 C_{\mathrm{T}}} \\
& +\frac{q_{\mathrm{T}}}{e}\left(c_{\mathrm{A}} \Phi^{\mathrm{A}}+c_{\mathrm{B}} \Phi^{\mathrm{B}}\right)
\end{aligned}
$$

At fixed $\left(q_{\mathrm{T}}, C_{i j}\right)$, the minimum energy

$$
W_{\mathrm{eq}}=-\frac{1}{2} C\left(\Delta_{\mathrm{A}}^{\mathrm{B}} \psi_{\mathrm{eq}}\right)^{2}+\frac{\left(q_{\mathrm{T}}\right)^{2}}{2 C_{\mathrm{T}}}+\frac{q_{\mathrm{T}}}{e}\left(c_{\mathrm{A}} \Phi^{\mathrm{A}}+c_{\mathrm{B}} \Phi^{\mathrm{B}}\right)
$$

corresponds to the equilibrium Volta potential difference $\Delta_{\mathrm{A}}^{\mathrm{B}} \psi_{\text {eq }}$, see eq 4. An external work $W-W_{\text {eq }}=C\left(\Delta_{\mathrm{A}}^{\mathrm{B}} \psi-\right.$ $\left.\Delta_{\mathrm{A}}^{\mathrm{B}} \psi_{\text {eq }}\right)^{2} / 2=\left(q_{\mathrm{eq}}^{\mathrm{B}}-q^{\mathrm{B}}\right)^{2} / 2 \mathrm{C}$ must be done to displace the system from equilibrium by transferring charge between the conductors.

If the conductors are two spheres of radii $r_{\mathrm{i}}(\mathrm{i}=\mathrm{A}, \mathrm{B})$, centerto-center distance $r_{\mathrm{A}}+r_{\mathrm{B}}+s$, and small separation $s$, the series expansions (up to order $s$ ) of the elements of the capacitance matrix are ${ }^{19}$

$$
\begin{aligned}
& C_{\mathrm{AA}}(s)=C_{0}\left[\lambda(s)-\psi_{0}\left(x_{\mathrm{B}}\right)\right] \\
& C_{\mathrm{BB}}(s)=C_{0}\left[\lambda(s)-\psi_{0}\left(x_{\mathrm{A}}\right)\right] \\
& C_{\mathrm{AB}}(s)=-C_{0}[\lambda(s)+\gamma]
\end{aligned}
$$

where $x_{\mathrm{i}}=r_{\mathrm{i}} /\left(r_{\mathrm{A}}+r_{\mathrm{B}}\right), C_{0} \equiv 4 \pi \varepsilon_{0} r_{\mathrm{A}} r_{\mathrm{B}} /\left(r_{\mathrm{A}}+r_{\mathrm{B}}\right), 2 \lambda(s)=$ $\ln \left\{2 r_{\mathrm{A}} r_{\mathrm{B}} /\left[\left(r_{\mathrm{A}}+r_{\mathrm{B}}\right) s\right]\right\}$, and $\psi_{0}(z)=\mathrm{d} \ln \Gamma(z) / \mathrm{d} z$ is the digamma function (defined as the logarithmic derivative of the gamma function, and not to be confused with the Volta potential $\psi$ ), and $\gamma=-\psi_{0}(1) \approx 0.5772$ is the Euler constant. Thus, the system capacitance, eq 5 , is

$$
C(s)=C_{0}\left[\lambda(s)+\frac{\gamma^{2}-\psi_{0}\left(x_{\mathrm{A}}\right) \psi_{0}\left(x_{\mathrm{B}}\right)}{\psi_{0}\left(x_{\mathrm{A}}\right)+\psi_{0}\left(x_{\mathrm{B}}\right)+2 \gamma}\right] \quad(s>0)
$$

and the outer potentials of the charged spheres are $\psi^{\mathrm{B}}=\Delta_{A}^{\mathrm{B}} \psi+$ $\psi^{\mathrm{A}}$ and

$$
\begin{aligned}
\psi^{\mathrm{A}} & =\psi_{\mathrm{av}}-\frac{C_{\mathrm{AB}}+C_{\mathrm{BB}}}{C_{\mathrm{AA}}+2 C_{\mathrm{AB}}+C_{\mathrm{BB}}} \Delta_{\mathrm{A}}^{\mathrm{B}} \psi \\
& =\psi_{\mathrm{av}}-\frac{\psi_{0}\left(x_{\mathrm{A}}\right)+\gamma}{\psi_{0}\left(x_{\mathrm{A}}\right)+\psi_{0}\left(x_{\mathrm{B}}\right)+2 \gamma} \Delta_{\mathrm{A}}^{\mathrm{B}} \psi
\end{aligned}
$$

The charges on the conductors can be calculated from these outer potentials. Such calculation requires eqs 3 and $8-11$ as the system capacitance depends strongly on the separation of the spheres when they are brought close to each other. It would be wrong to consider that the spheres have the same capacitance when isolated in vacuum.

Consider two uncharged $\left(q_{\mathrm{T}}=0\right.$ and $\left.\psi_{\mathrm{av}}=0\right)$ large spheres of silver $\left(\Phi^{\mathrm{Ag}}=4.30 \mathrm{eV}\right)$ and gold $\left(\Phi^{\mathrm{Au}}=5.30 \mathrm{eV}\right) .{ }^{20}$ Upon contact, the electrons flow from silver to gold to generate a Volta potential difference $\psi^{\mathrm{Ag}}-\psi^{\mathrm{Au}}=1.00 \mathrm{~V}$ and equalize the Fermi levels. From eq 12, if the spheres have equal radii, $x_{\mathrm{A}}=x_{\mathrm{B}}$ $=1 / 2$, then $\psi^{\mathrm{Ag}}=-\psi^{\mathrm{Au}}=-\Delta_{\mathrm{Ag}}^{\mathrm{Au}} \psi / 2=0.50 \mathrm{~V}$ and the Fermi level of the spheres in contact is the average of the two Fermi levels, that is, $4.80 \mathrm{eV}$. Figure 1a illustrates schematically the potential distribution. After equilibration, the electrochemical potential of the electrons is the same in the two spheres (Figure 1b). This indicates that the work to remove one electron (from the interior to infinity) has to be the same for both spheres. Although the surface charge distribution is not uniform on the spheres, the outer potential of each sphere is constant, and hence the energy required to extract the electron from the interior through any surface point is the same for all surface 
points. Similarly, if we consider a charged cube of a conducting material in vacuum, the excess surface charge is distributed to have a constant potential around the cube with most of it located at the corners of the cube. ${ }^{26,27}$ The system has to be in thermodynamic equilibrium, so the work to remove one electron through a corner is the same as the work to remove it through the middle of the side. ${ }^{26,27}$

If the radius of the silver sphere is, say, 1000 times larger (Figure $1 \mathrm{c}$ ), then $x_{\mathrm{A}}=1-x_{\mathrm{B}} \approx 1$ and eq 12 predicts $\psi^{\mathrm{Ag}} \approx 0$, that is, the Fermi level of the equilibrated system is close to that of uncharged, pure silver (Figure 1d). Although the absolute charges on both spheres are the same, the small gold sphere acquires a high (negative) surface charge density so that $\psi^{\mathrm{Au}} \approx$ $-1.00 \mathrm{~V}$ and its Fermi level increases by $1.00 \mathrm{eV}$, whereas the larger silver sphere has a negligible surface charge density and $\psi^{\mathrm{Ag}} \approx 0$. If we separate the spheres, we have a charged gold sphere and a negligibly charged silver sphere. The gold sphere is electron rich and this may have interesting applications, for example, in plasmonics. ${ }^{28-30}$ On the contrary, if the gold sphere is much larger than the silver sphere, then the equilibrated Fermi level is that of uncharged gold and the silver is positively charged. The variation in the potentials of the spheres with their relative size is presented in Figure 2 for the case $q_{\mathrm{T}}=0$ and $\psi_{\mathrm{av}}=0$.

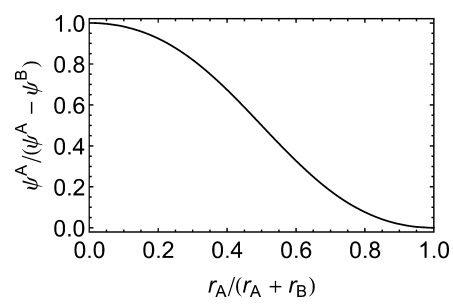

Figure 2. $\psi^{\mathrm{A}} /\left(\psi^{\mathrm{A}}-\psi^{\mathrm{B}}\right)$ as a function of $r_{\mathrm{A}} /\left(r_{\mathrm{A}}+r_{\mathrm{B}}\right)$ showing that when $r_{\mathrm{A}} \ll r_{\mathrm{B}}$, the potential difference is practically equal to that between the smaller one and vacuum, while the bigger one has practically zero potential, as described schematically in Figure 1d.

The total magnitude of the transferred charge depends on the size of the contact interface. For example, a total charge transfer of $4 \times 10^{6}$ electrons $(0.6 \mathrm{pC})$ is observed between a $5 /$ 32 in. diameter chromium sphere and a $1 / 2$ in. diameter gold sphere, ${ }^{16}$ whereas DFT calculations done with 591 different atom $\mathrm{Au}-\mathrm{Ag}$ particles show total charge transfer of 5-17 electrons, depending on the arrangement of the atoms. ${ }^{18}$ These examples show that the relative amount of the transferred electrons can be very high in nanoscale systems, whereas less significant effects are observed in the macroscopic systems.

Force between the Metallic Spheres. The rate of variation in the system energy, eq 6, with the separation $s$ of the spheres provides the force between them. Equations 8-10 imply that $c_{\mathrm{A}}, c_{\mathrm{B}}$, and $C_{\mathrm{T}}$ are independent of $s$, and that the system capacitance decreases with increasing $s$

$$
\frac{\mathrm{d} C}{\mathrm{~d} s}=\frac{\mathrm{d} C_{\mathrm{AA}}}{\mathrm{d} s}=\frac{\mathrm{d} C_{\mathrm{BB}}}{\mathrm{d} s}=-\frac{\mathrm{d}_{\mathrm{AB}}}{\mathrm{d} s}=-\frac{C_{0}}{2 s}
$$

When the conductors are separated without charge transfer, the quantity $C \Delta_{\mathrm{A}}^{\mathrm{B}} \psi=c_{\mathrm{A}} q^{\mathrm{B}}-c_{\mathrm{B}} q^{\mathrm{A}}$ remains constant, but $C$ varies with $s$ as does $\Delta_{A}^{\mathrm{B}} \psi$, unless $\Delta_{\mathrm{A}}^{\mathrm{B}} \psi=0$. Thus, from eq 6 , the force between the two spheres under this constraint is

$$
\begin{gathered}
F_{q^{\mathrm{A}}, q^{\mathrm{B}}}=-\left(\frac{\partial W}{\partial s}\right)_{q^{\mathrm{A}}, q^{\mathrm{B}}}=-C \Delta_{\mathrm{A}}^{\mathrm{B}} \psi \frac{1}{2}\left(\frac{\partial \Delta_{\mathrm{A}}^{\mathrm{B}} \psi}{\partial C}\right)_{C \Delta_{\mathrm{A}}^{\mathrm{B}} \psi} \frac{\mathrm{d} C}{\mathrm{~d} s} \\
=-\frac{1}{4}\left(\Delta_{\mathrm{A}}^{\mathrm{B}} \psi\right)^{2} \frac{C_{0}}{s}
\end{gathered}
$$

Similarly, the force between the conductors when they are separated at a constant potential difference (by allowing charge transfer between the conductors by electron tunneling) is

$$
F_{q_{\mathrm{T}}, \Delta_{\mathrm{A}}^{\mathrm{B}} \psi}=-\left(\frac{\partial W}{\partial C}\right)_{q_{\mathrm{T}}, \Delta_{\mathrm{A}}^{\mathrm{B}} \psi} \frac{\mathrm{d} C}{\mathrm{~d} s}=\Delta_{\mathrm{A}}^{\mathrm{B}} \psi\left(\frac{1}{e} \Delta_{\mathrm{A}}^{\mathrm{B}} \Phi+\frac{1}{2} \Delta_{\mathrm{A}}^{\mathrm{B}} \psi\right) \frac{C_{0}}{2 s}
$$

If two spheres of different materials are separated after equilibration of their Fermi levels, the force between them depends on the constraint imposed during the separation. At constant charge, the Volta potential difference increases with increasing separation from its initial value, $\left|\Delta_{A}^{\mathrm{B}} \psi\right| \geq\left|\Delta_{A}^{\mathrm{B}} \psi_{\mathrm{eq}}\right|$, and hence the force is more attractive than at the constant potential difference

$$
F_{q_{\mathrm{eq}}^{\mathrm{A}}, q_{\mathrm{eq}}^{\mathrm{B}}}=-\frac{1}{2}\left(\Delta_{\mathrm{A}}^{\mathrm{B}} \psi\right)^{2} \frac{C_{0}}{2 s} \leq-\frac{1}{2}\left(\Delta_{\mathrm{A}}^{\mathrm{B}} \psi_{\mathrm{eq}}\right)^{2} \frac{C_{0}}{2 s}=F_{q_{\mathrm{T}}}, \Delta_{\mathrm{A}}^{\mathrm{B}} \psi_{\mathrm{eq}}
$$

The Volta potential difference during and after contact of two spheres of the same material is $\Delta_{A}^{\mathrm{B}} \psi=0$, and hence eq 14 predicts that the force $F_{q^{A}}, q^{B}$ vanishes. However, this conclusion is based on approximate expressions for $c_{\mathrm{A}}, c_{\mathrm{B}}$, and $C_{\mathrm{T}}$, which makes them independent of the separation $s$. When higher order terms are included in the series expansions of the elements of the capacitance matrix, then $F_{q^{\mathrm{A}}, q^{\mathrm{B}}}$ turns out to be repulsive. ${ }^{17}$

If the spheres are close enough to equilibrate the Fermi levels (i.e., electron tunneling is possible), the potential difference $\Delta_{\mathrm{A}}^{\mathrm{B}} \psi_{\mathrm{eq}}=-\Delta_{\mathrm{A}}^{\mathrm{B}} \Phi / e$ is constant. However, if the electron tunneling is not possible, the charge is then constant. So if we have ideal spheres of two different metals without any surface roughness, this result indicates that when they are in contact, the Fermi levels equilibrate and the force between them is attractive. If the spheres are separated, there is some transferred charge that tries to keep the potential difference constant, that is, to maintain the condition $\tilde{\mu}_{\mathrm{e}^{-}}^{\mathrm{A}}=\tilde{\mu}_{\mathrm{e}^{-}}^{\mathrm{B}}$. When the cutoff distance of the electron tunneling is reached, the charge remains constant and the attractive force is then given by eq 12 with $\Delta_{\mathrm{A}}^{\mathrm{B}} \psi$ varying with $s$. If $q_{\mathrm{T}}=0$, because the conductors have opposite charges $q^{\mathrm{B}}=-q^{\mathrm{A}}=C \Delta_{\mathrm{A}}^{\mathrm{B}} \psi$, then eqs 11 and 14 allow to write the attractive force between the conductors when separated at constant charge as

$$
\begin{aligned}
F_{q^{\mathrm{A}}, q^{\mathrm{B}}} & =-\frac{C_{0}}{4 s}\left(\Delta_{\mathrm{A}}^{\mathrm{B}} \psi\right)^{2} \\
& =-\frac{\left(q^{\mathrm{B}}\right)^{2}}{4 C_{0} s}\left[\lambda(s)+\frac{\gamma^{2}-\psi_{0}\left(x_{\mathrm{A}}\right) \psi_{0}\left(x_{\mathrm{B}}\right)}{\psi_{0}\left(x_{\mathrm{A}}\right)+\psi_{0}\left(x_{\mathrm{B}}\right)+2 \gamma}\right]^{-2}
\end{aligned}
$$

\section{CHARGING OF BIMETALLIC JANUS PARTICLES}

Fermi Level Equilibrium. Consider two uncharged metals $A$ and $B$ in vacuum, each made of two half-spheres linked by a wire of the same metal (Figure 3a). Because no electric field exists outside of the metals, two positions A2 and B2 just outside of the metals have the same potential $\psi^{\mathrm{A} 2}=\psi^{\mathrm{B} 2}$. The 

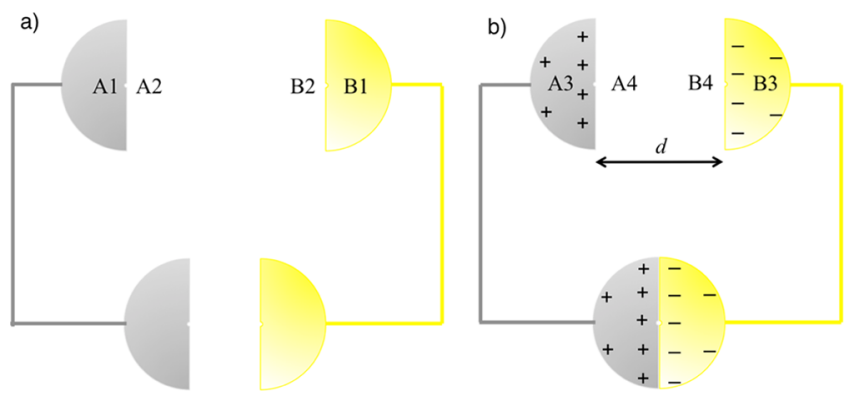

Figure 3. (a) Two uncharged metals with different work functions in vacuum. (b) Contact electrification equilibrates their Fermi levels. The signs + and - correspond to $\Phi^{\mathrm{B}}>\Phi^{\mathrm{A}}$.

metals are then brought in contact through the lower hemispheres and reach the distribution equilibrium condition $\tilde{\mu}_{\mathrm{e}^{-}}^{\mathrm{A}}=\tilde{\mu}_{\mathrm{e}^{-}}^{\mathrm{B}}$. The charge redistribution that leads to the equilibration of the Fermi levels affects the outer potentials of the charged phases but not their work functions. Two positions A4 and B4 just outside of the metals (Figure $3 \mathrm{~b}$ ) have a potential difference given by eq $6, \psi^{\mathrm{B4}}-\psi^{\mathrm{A} 4}=\psi^{\mathrm{B}}-\psi^{\mathrm{A}}$. As the Galvani potential $\phi=\chi+\psi$ is constant within a phase at equilibrium (see the Supporting Information), the Volta potential difference equals the sum of the potential drops between positions $\mathrm{B} 4$ and $\mathrm{B} 3$, the potential drop across the plane of contact of the lower hemispheres, and the potential drop between positions A3 and A4. The surface potential $\chi$ at a metal-vacuum interface is not affected by the charge on the metal, ${ }^{31}$ and therefore the potential drops between positions B3 and $\mathrm{B} 4$ and between positions $\mathrm{A} 3$ and $\mathrm{A} 4$ are $\chi^{\mathrm{B}}$ and $\chi^{\mathrm{A}}$, respectively. Then, the Galvani potential difference between $\mathrm{A}$ and $\mathrm{B}$ is

$$
\begin{aligned}
\left(\phi^{\mathrm{B}}-\phi^{\mathrm{A}}\right)_{\text {contact }} & =\left(\mu_{\mathrm{e}^{-}}^{\mathrm{B}}-\mu_{\mathrm{e}^{\mathrm{A}}}^{\mathrm{A}}\right) / e \\
& =\chi^{\mathrm{B}}-\chi^{\mathrm{A}}+\left(\Phi^{\mathrm{A}}-\Phi^{\mathrm{B}}\right) / e
\end{aligned}
$$

Across a few atomic layers at the plane of contact of the lower hemispheres, a surface density of dipoles $\sigma_{\text {dip }}$ creates this difference of inner potentials $\left(\phi^{\mathrm{B}}-\phi^{\mathrm{A}}\right)_{\text {contact. }}$

A common belief about metal-metal contacts is that all the charges transferring from $\mathrm{A}$ to $\mathrm{B}$ are located at the contact itself, as interfacial dipoles that form polarized covalent bonds between the interfacial atoms. This, however, is not completely true as some charges spread all over the outer surfaces of the metal to induce the electric potential in the metal, and this charge distribution is dictated by the capacitance of the whole system. For example, the simple electrostatic model predicts that ca. $10 \%$ of the transferred charge is actually located on the outer surfaces of a $12.6 \AA$ radius Janus particle (vide infra), but this ratio is expected to depend strongly on the geometry of the system.

The total capacitance of the system shown in Figure $3 \mathrm{~b}$ can be considered as the sum of the capacitance of the upper and lower hemispheres (as the system is equivalent to two capacitors in parallel). This simple electrostatic model for the contact electrification of Janus particles has been justified by comparison with DFT calculations. ${ }^{18}$ The electrons in both phases have the same electrochemical potential. This means that the difference in real potential of electrons in different metals is compensated by a Volta potential difference between the metals. If we describe the metal-metal contact as a planar capacitor and the surface density of dipoles as $\sigma_{\text {dip }}=\sigma_{\mathrm{c}} d_{\mathcal{c}}$ then

$$
\left(\phi^{\mathrm{B}}-\phi^{\mathrm{A}}\right)_{\text {contact }}=Q_{\mathrm{c}} / C_{\mathrm{c}}=\sigma_{\mathrm{c}} d_{\mathrm{c}} / \varepsilon_{0}
$$

where the subscript $\mathrm{c}$ stands for contact and $\varepsilon_{0}$ is the vacuum permittivity. Similarly, if we consider that the upper hemispheric pieces form a pseudoplanar capacitor and neglect the edge effect, we have

$$
\psi^{\mathrm{B} 4}-\psi^{\mathrm{A} 4}=\psi^{\mathrm{B}}-\psi^{\mathrm{A}}=\sigma d / \varepsilon_{0}
$$

where $d$ is the gap of the capacitor and $\sigma$ the surface charge density on the two opposing metal faces. If we assume that the difference in surface potential is very small, eqs $18-20$ imply that $\sigma_{\mathrm{c}} d_{\mathrm{c}}=\sigma d$, which means that most of the transferred charge is at the contact plane because $d_{c} \ll d$. That is, the surface charge density at the place of contact is much higher than that in the separated hemispheres because the distance between the plates is much smaller. In addition, a small amount of charge resides on the outer surfaces of the metal, as each metal has uniform potential.

In an ideal $\mathrm{Ag}-\mathrm{Au}$ Janus sphere formed by contact of two equal size hemispheres, the electrons flow from silver to gold to equalize the Fermi levels because $\Phi^{\mathrm{Ag}}=4.30 \mathrm{eV}$ and $\Phi^{\mathrm{Au}}=5.30$ $\mathrm{eV}^{20}$ By symmetry, the Fermi level of this ideal Janus sphere is the average of the Fermi levels of the two metals before contact, that is, $4.80 \mathrm{eV}$. The Volta potential difference $\psi^{\mathrm{Ag}}-\psi^{\mathrm{Au}}=1.00$ $\mathrm{V}$ is given by eq 4 , that is, by the common IE, IE $=\Phi^{\mathrm{Ag}}+e \psi^{\mathrm{Ag}}=$ $\Phi^{\mathrm{Au}}+e \psi^{\mathrm{Au}}$. Figure 4 illustrates schematically the potential distribution on the symmetry axis.

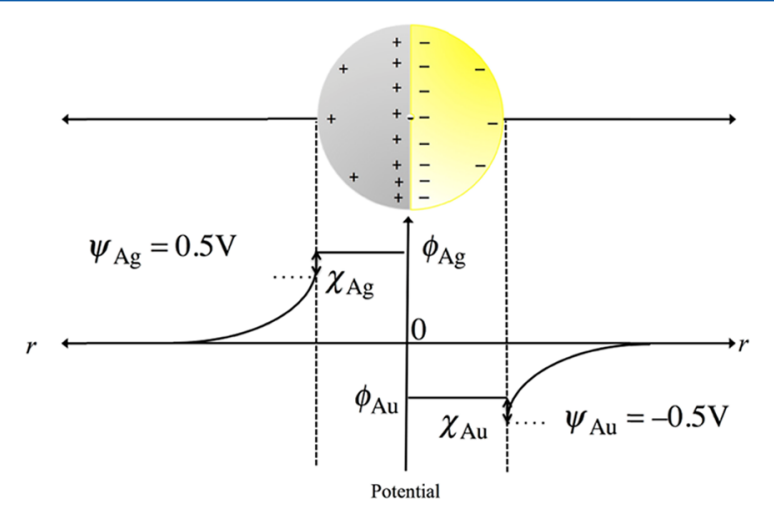

Figure 4. Potential distribution along the equatorial axis for a $\mathrm{Ag}-\mathrm{Au}$ Janus sphere. $\chi^{\mathrm{Ag}}$ is not necessarily equal to $\chi^{\mathrm{Au}}$.

Let us consider now what happens with the Fermi level when the surface fraction of the metals changes (Figure 5). This problem was rigorously studied by Langmuir, who considered the effect of surface coverage of Cs on tungsten on the work function. ${ }^{32}$ Alkali metal atoms adsorbed on various other metals show relatively similar trends: a rapid decrease in the work function with the increasing alkali metal coverage followed by a broad minimum. As the coverage approaches unity, the work function increases again and levels off at the work function of the alkali metal. ${ }^{33}$ This behavior can be explained by the fact that at low coverages alkali metal atoms are present as single ions leading to the formation of strong dipoles, whereas on increasing the surface coverage this polarization decreases because of the stronger interaction between dipoles and the formation of covalent bonds between adsorbates. In effect, the coordination number of atoms increases, and finally the surface layer starts to resemble the bulk materials. ${ }^{33}$ Interestingly, these mixtures produce materials with lower work functions than 

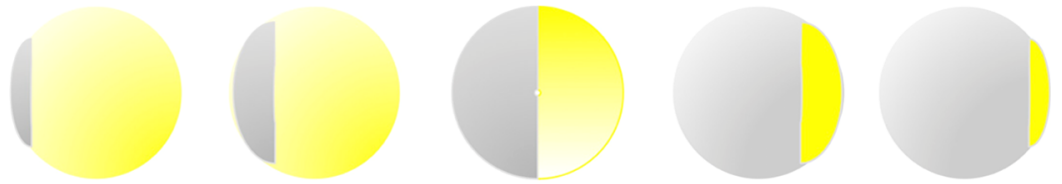

Figure 5. The variation in the surface fraction of silver in $\mathrm{Ag}-\mathrm{Au}$ Janus particles.
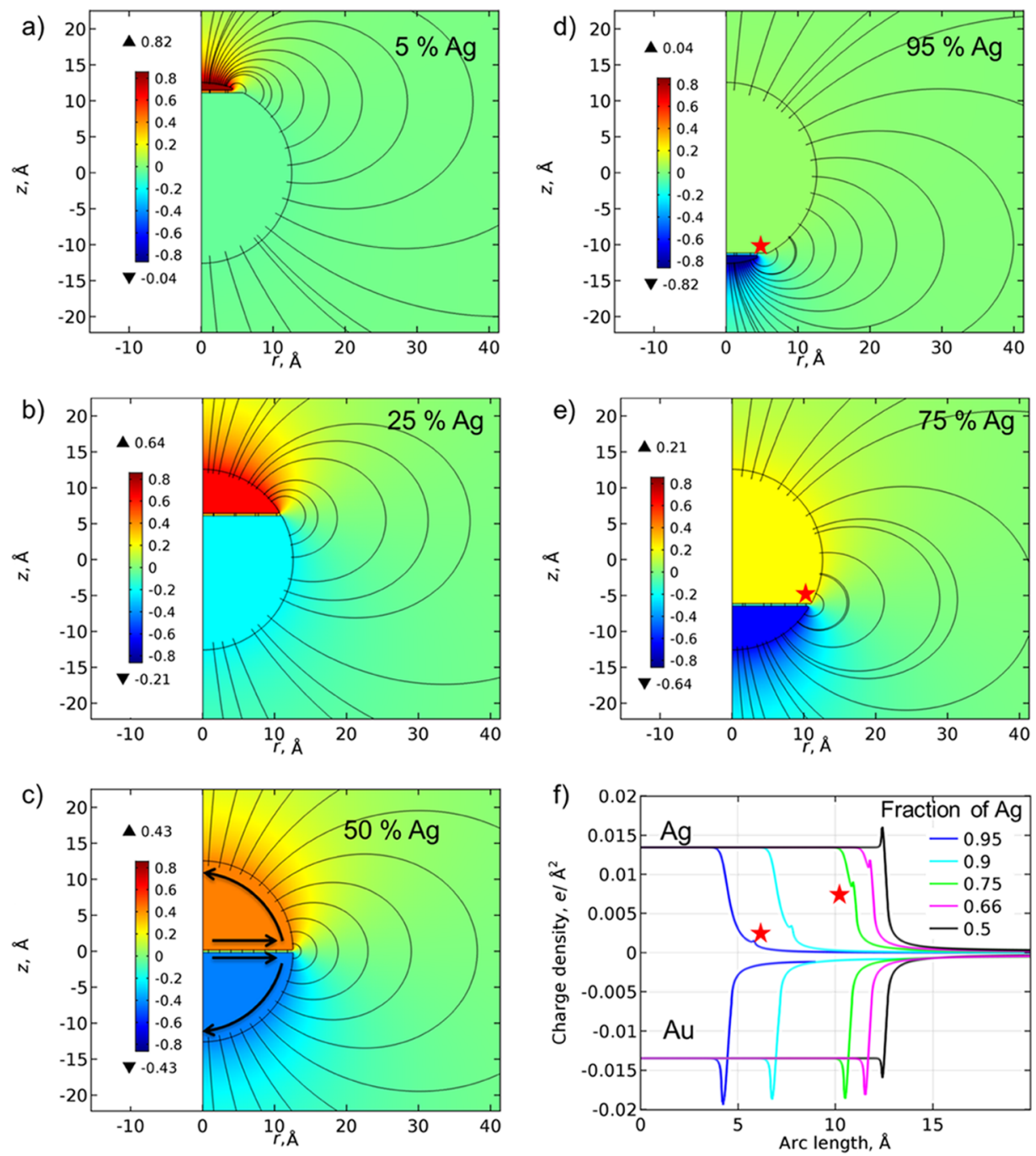

Figure 6. (a-e) The potential distributions, shown in $2 \mathrm{D}$ axial symmetry, of spherical $\mathrm{Ag}-\mathrm{Au}$ Janus particles with different surface fractions of $\mathrm{Ag}$ (see Figure 5; note that a surface fraction $x_{\mathrm{s}}^{\mathrm{Ag}}=5 \%$ corresponds to a volume fraction $\left(3-2 x_{\mathrm{s}}^{\mathrm{Ag}}\right)\left(x_{\mathrm{s}}^{\mathrm{Ag}}\right)^{2}=0.725 \%$ ). The silver cap has a positive charge. (f) Surface charge density as a function of the arc length as shown in (c). The red stars mark the position of the shoulder in (d) and (e).

both bulk materials. Recently, the additional effect of the orbital overlap between both metals has also been highlighted. ${ }^{34}$ However, for fully coordinated surfaces, the formation of such dipoles is suppressed. ${ }^{34}$ In this case, we have recently shown that the Fermi level of the silver-gold system is directly dependent on the surface area ratio between the two metals, whereas there was no influence on the Fermi level by the volume or molar ratio of the two elements. ${ }^{18}$ This relation is expected to hold also for other systems where metals at the surface are bound by metal-metal bonds and segregated into large enough clusters.

In fact, the Fermi level $E_{\mathrm{F}}$ of these systems can be approximated by

$$
E_{\mathrm{F}}^{\mathrm{Au}-\mathrm{Ag}}=x_{\mathrm{s}}^{\mathrm{Au}} E_{\mathrm{F}}^{\mathrm{Au}}+x_{\mathrm{s}}^{\mathrm{Ag}} E_{\mathrm{F}}^{\mathrm{Ag}}
$$

where $x_{\mathrm{s}}^{\mathrm{Au}}=1-x_{\mathrm{s}}^{\mathrm{Ag}}$ is the surface area fraction of Au. This relationship in the case of polycrystalline surfaces is considered to determine the average work function of the surface as the weighted average of the work functions of the individual crystallites known as "patches". ${ }^{35,36}$ In the case of planar, cylindrical, and spherical surfaces, the average work function is the surface area-weighted average of the work functions of the individual patches. ${ }^{35,36}$ For more complex geometries, the electrostatic interactions between oppositely charged patches have to be considered more carefully as described, for example, by Sahni et al. ${ }^{35}$ and Baldereschi et al., ${ }^{26}$ and also in this work for two spheres. Similar considerations apply to the surfaces covered with patches of different metals, but in this case the Fermi level differences between the two metals can be much higher, of the order of electronvolts, leading to higher surface 
charge densities and stronger electric fields than in the case of surfaces of a single metal. Recently, this relationship has been illustrated for different bimetallic $\mathrm{Ag}-\mathrm{Au}$ nanoparticles containing 591 atoms on $\mathrm{Au}-\mathrm{Ag}$ surfaces and on $\mathrm{Au}$ nanoislands on the Ag surface. ${ }^{18}$

The case described is very similar to the formation of alloys, where two components are homogeneously mixed. The work function and the Fermi level of alloys have been considered both theoretically and experimentally. Computational methods and theoretical correlations based on image force, dipole layer, surface mixture, and dual phase mixture approaches have been proposed. ${ }^{37}$ In the case of contact electrification, the situation is much simpler than with alloys, because only one surface layer is affected, whereas in alloys the components can be completely mixed. Alloying changes the Fermi level in two ways: by contact electrification, as described above, and by changing the lattice structure of the metal, directly affecting the chemical potential of electrons in the alloy. If the lattice structure does not change significantly, the Fermi level of alloys can also be estimated with eq 21.

Contact Charging in Vacuum. The evaluation of the amount of transferred charge is a complicated issue, as it strongly depends on the geometry of the contact interface. To illustrate this, charge distribution upon contact charging was investigated with the capacitance model. ${ }^{18}$ The Laplace equation $\nabla^{2} \phi=0$ for the electrostatic potential in vacuum was solved in 2D axis symmetry, both analytically and numerically using COMSOL Multiphysics, to illustrate the potential distribution because of the contact electrification of $\mathrm{Ag}-\mathrm{Au}$ Janus particles (radius $12.6 \AA$ ) with different compositions (see the Supporting Information for details). In particular, the surface fraction of Ag was varied from 5 to $95 \%$ (Figure 5). All computations were performed using a MacIntosh computer with 4 Intel Xeon(R) 5150 processors operating at $2.66 \mathrm{GHz}$ and using the Windows 7 operating system; the runtimes were up to $1 \mathrm{~min}$.

We have recently shown that the metals in contact can be considered as two electrodes of a capacitor separated by the tiny gap. ${ }^{18}$ The separation between the two metals was chosen as $0.353 \AA$ so that the charge transferred matched the value obtained by DFT calculations. ${ }^{18}$ The results are shown in Figure 6 with streamlines for the electric field. In this case, the potential difference between the spherical caps is equal to the difference in the Fermi levels of the pure metal nanoparticles $(0.86 \mathrm{~V}$ as calculated for icosahedral nanoparticles of 591 atoms). ${ }^{18}$

The simulations show that ca. $10 \%$ of the transferred charge is on the outside, whereas $90 \%$ is retained at the contact interface. The surface charge density is shown in Figure $6 \mathrm{f}$. The electric field is the strongest in the gap and vanishes almost completely five particle radii away from the sphere. Although these calculations are simplifications of the geometry of the system, they allow understanding the magnitude of the surface charge density. The small bumps on the surface charge of silver and a higher peak for the gold in Figure 6e are located at the three-phase boundary between silver, gold, and the surrounding medium at the outer surface. This is because the boundary between the surface of the sphere and the gold cap has a sharper edge. Simulations done with surface fractions of 33, 25, 10, and 5\% of $\mathrm{Ag}$ show the same behavior, but with inversed charge density profiles (Ag showing the peak), as expected by symmetry.
Potential Distribution around Bimetallic Janus Nanoparticles in Electrolyte Solution. When a material bearing a surface charge is placed into an electrolyte solution, an ionic double layer forms around the material, effectively screening the surface charge. Because we want to understand what happens when contact charging takes place in aqueous electrolyte solutions, a brief overview of the electric double layer, the Poisson-Boltzmann model, and their modifications is given in this section. The electric double layer forms on all charged interfaces in contact with electrolyte solutions. The charge confined on the surface affects the solvent molecules and the ions in the electrolyte solution. The generally accepted model of the electric double layer contains inner and outer Helmholtz layers and a diffuse double layer. The outer Helmholtz layer is the closest approach of solvated ions to the surface, whereas the inner Helmholtz layer consists of mostly organized solvent dipoles and may also contain some specifically adsorbed ions that have lost their solvation shell. Sometimes the inner and outer Helmholtz planes are together called as a Stern layer. ${ }^{20}$

In a binary symmetric electrolyte $\left(z_{+}=\left|z_{-}\right|=z\right)$, the Poisson-Boltzmann equation is

$$
\varepsilon_{r} \varepsilon_{0} \nabla^{2} \phi=2 z \mathrm{Fc}^{\mathrm{b}} \sinh \left(\frac{z \mathrm{~F} \phi}{R T}\right)
$$

where $c_{+}^{\mathrm{b}}=c_{-}^{\mathrm{b}}=c^{\mathrm{b}}$ is the molar concentration of the ionic species at the bulk of the solution (where $\phi=0$ ). As it is well known from the Gouy-Chapman description of the electric double layer, a limitation of this model is that the ions are considered as point charges, resulting in abnormally high surface concentrations of ions at high polarizations. One solution proposed by Cervera and co-workers takes into account the steric effects in the Boltzmann distribution, ${ }^{38-42}$ leading to the modified Boltzmann distribution as follows:

$$
c_{\mathrm{i}}=\frac{c_{\mathrm{i}}^{\mathrm{b}} \exp \left(-\frac{z_{\mathrm{i}} \mathrm{F} \phi}{R T}\right)}{1+2 v_{\mathrm{i}} \sinh ^{2}\left(\frac{z F \phi}{2 R T}\right)}
$$

where $v_{\mathrm{i}}$ is a packing parameter expressed as $v_{\mathrm{i}}=2 N_{\mathrm{A}} a_{\mathrm{i}}^{3} c_{\mathrm{i}}^{\mathrm{b}}, N_{\mathrm{A}}$ is Avogadro's constant, and $a_{\mathrm{i}}$ is the diameter of the solvated ion $\mathrm{i}$. Similar expressions were proposed in the 1940s and 1950s by Bikerman, Grimley, and Mott, Eigen and Wicke, Freise, and Brodowsky and Strehlow as reviewed in refs 39 and 42. The modified Poisson-Boltzmann equation then becomes

$$
\varepsilon_{r} \varepsilon_{0} \nabla^{2} \phi=\frac{2 z F c^{\mathrm{b}} \sinh \left(\frac{z F \phi}{R T}\right)}{1+2 v_{\mathrm{i}} \sinh ^{2}\left(\frac{z F \phi}{2 R T}\right)}
$$

This modification ensures that the surface ionic concentrations reach a saturation point at the steric limit with increasing surface polarization, whereas the unmodified PoissonBoltzmann equations predict continuous increase in concentrations. Although eq 24 flattens out any oscillations in the charge density distribution because of finite-size effects, ${ }^{42}$ these oscillations are not expected to be important at the charge densities owing to the contact electrification.

The Stern modification of the Gouy-Chapman model adds the inner and outer Helmholtz planes into the system. ${ }^{20}$ Because there are no ions within the Stern layer (if the specific ion adsorption is not considered), the electrostatic potential satisfies the Laplace equation $\nabla^{2} \phi=0$. This equation is also 
valid in any phase where there are no ions and dipoles, and hence there is no formation of the electric double layer.

To evaluate the effect of the electrolyte on the potential distribution around a bimetallic particle, simulations were done in $10 \mathrm{mM} \mathrm{KCl}$ using the modified Poisson-Boltzmann equation 24 with a Stern layer of thickness of $3.3 \AA$ (roughly the radius of a hydrated $\mathrm{K}^{+}$or $\mathrm{Cl}^{-}$ion ${ }^{43}$ ). For simplicity, the relative permittivity in this Stern layer was considered as the bulk value of water, $\varepsilon_{\mathrm{r}}=78$. More accurate simulations could be obtained by using the Booth model to account for the electric field-dependent permittivity of water in the Stern layer. ${ }^{44-46}$ The potential profiles obtained by solving the modified Poisson-Boltzmann equation are very similar to those in vacuum (Figure 6), so only the potential profile of the $50 \%$ $\mathrm{Ag}-\mathrm{Au}$ Janus particle is shown in Figure $7 \mathrm{a}$. About half of the potential drop occurs in the Stern layer, and the electric field almost completely disappears beyond the Debye length (calculated as $33 \AA$ for $10 \mathrm{mM} \mathrm{KCl}$ ). Figure $7 \mathrm{~b}$ shows the

a)
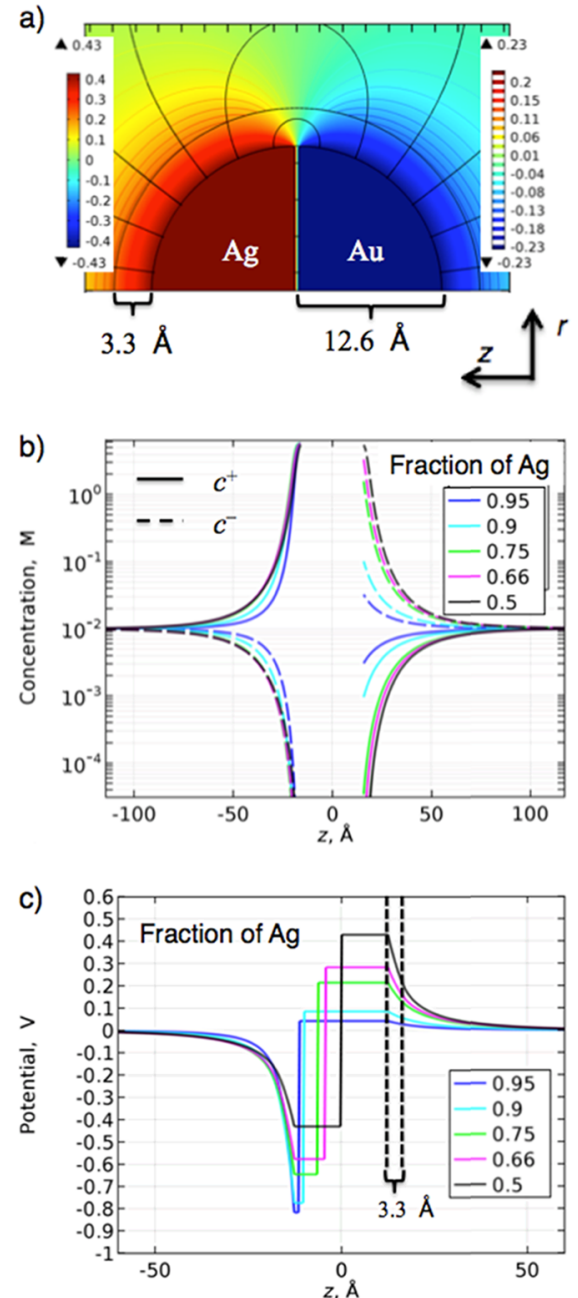

Figure 7. (a) The potential distributions around a spherical $\mathrm{Ag}-\mathrm{Au}$ Janus particle of $12.6 \AA$ radius immersed in a $10 \mathrm{mM} \mathrm{KCl}$ aqueous solution $\left(\varepsilon_{\mathrm{r}}=78\right)$, shown in $2 \mathrm{D}$ axial symmetry. A Stern layer of $3.3 \AA$ has been considered. The potentials in the diffuse double layer vary from 0.23 to $-0.23 \mathrm{~V}$. (b) Concentration profiles of cations and anions in the electric double layer along the $z$ axis for different Ag surface fractions. (c) Potential profiles along the $z$ axis for different Ag surface fractions. The dashed lines indicate the thickness of the Stern layer on the Ag surface. concentration profiles of cations (solid line) and anions (dashed line) along the $z$ axis. The cases with 75,66 , and $50 \%$ surface fractions of $\mathrm{Ag}$ are almost indistinguishable from each other on the Au side, whereas stronger variation is observed on the $\mathrm{Ag}$ side because of lower potentials. This is emphasized in Figure $7 c$, where potential of Au decreases with increasing $\mathrm{Ag}$ surface fraction. The potential profiles are inversed for smaller Ag fractions, as expected by symmetry.

\section{FERMI LEVEL EQUILIBRATION IN CORE-SHELL PARTICLES}

Let us consider a gold sphere coated with a silver layer (Figure 8) with no net charge and hence zero outer potential. The

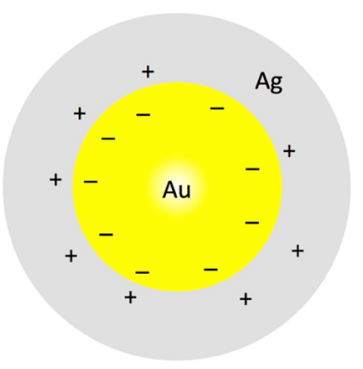

Figure 8. Au@Ag core-shell nanoparticle.

Fermi level of the silver shell is the same as that of a pure, uncharged silver particle. If we move a test charge from vacuum into the shell of $\mathrm{Ag}$, we have to overcome the surface potential $\chi^{\mathrm{Ag}}$ between the $\mathrm{Ag}$-vacuum interface and also consider the interactions between electrons and atoms in the Ag phase, $\tilde{\mu}_{\mathrm{e}^{-}}^{\mathrm{Ag}}$, giving the total work $\tilde{\mu}_{\mathrm{e}^{-}}^{\mathrm{Ag}}=\tilde{\mu}_{\mathrm{e}^{-}}^{\mathrm{Ag}}-e \chi^{\mathrm{Ag}}$ (note that there is no outer surface charge and $\psi^{\mathrm{Ag}}=0$ ). The work function of the core-shell particle is the same as for the pure outer metal, $\mathrm{IE}^{\mathrm{Au} @ \mathrm{Ag}}=-\tilde{\mu}_{\mathrm{e}^{-}}^{\mathrm{Ag}}+e \chi^{\mathrm{Ag}}=\Phi^{\mathrm{Ag}}$, except for the strain induced by the mismatch of the lattice constants of the two metals, affecting the real potential of electrons in the outer metal. The charge distribution at the interface between the two metals, which results from the electron flow (and bond polarization) from $\mathrm{Ag}$ to $\mathrm{Au}$, makes the Fermi level of the $\mathrm{Au}$ core to be the same as that of the Au shell. The Galvani potential difference $\Delta_{\mathrm{Au}}^{\mathrm{Ag}} \phi=\phi^{\mathrm{Ag}}-\phi^{\mathrm{Au}}$ is created by the charge distribution at this interface. To evaluate the electrochemical potential of the electrons in the $\mathrm{Au}$ core, the test charge has to be moved through the $\mathrm{Ag}-\mathrm{Au}$ interface, resulting in $\tilde{\mu}_{\mathrm{e}^{-}}^{\mathrm{Au}}=\tilde{\mu}_{\mathrm{e}^{-}}^{\mathrm{Ag}}=\tilde{\mu}_{\mathrm{e}^{-}}^{\mathrm{Au}}-e \chi^{\mathrm{Ag}}-e \Delta_{\mathrm{Au}}^{\mathrm{Ag}} \phi$.

The surface density of dipoles that generates the Galvani potential difference can be estimated as $\sigma_{\text {dip }}^{\mathrm{Au} @ \mathrm{Ag}} / \varepsilon_{0}=\phi^{\mathrm{Ag}}-\phi^{\mathrm{Au}}=\left(\tilde{\mu}_{\mathrm{e}^{-}}^{\mathrm{Au}}-\tilde{\mu}_{\mathrm{e}^{-}}^{\mathrm{Ag}}\right) / e$. If the magnitude of this Galvani potential difference is estimated roughly as the work function difference of pure metals, the surface dipole density will be $\sigma_{\text {dip }}^{\mathrm{Au} @ \mathrm{Ag}}=8.8 \times 10^{-12} \mathrm{C} / \mathrm{m}$. The charge separation in metals occurs over a distance of the order of the Thomas-Fermi length $\left(r_{\mathrm{TF}} \approx 0.05 \mathrm{~nm}\right)$, and therefore this charge density of dipoles corresponds to $\sigma_{\mathrm{dip}}^{\mathrm{Au} @ \mathrm{Ag}} / r_{\mathrm{TF}}=0.17 \mathrm{C} /$ $\mathrm{m} \approx 1 \mathrm{e} / \AA^{2}$ showing that the charge at the interfacial dipole depends on the radius of the core (as the surface area of the interface depends on the radius) but not on the thickness of the shell. Of course, there are additional factors like the surface stress that also influence the Fermi level of electrons in the 
core-shell particles, but the general principles described here apply.

Core-shell particles are known to have markedly different optical properties than the pure metal nanoparticles, and the experimental spectra can be reasonably reproduced theoretically by using Mie theory for multilayer concentric spheres. ${ }^{47,48}$ The general observation is that when the size of the shell grows, the plasmon resonance of the core is quickly masked by the response from the shell and only at certain shell thicknesses the two plasmon resonances are present. ${ }^{47}$ Although it is well known that charging of the nanoparticle can have a significant effect on the plasmon resonance, ${ }^{47,49,50}$ the effect of contact charging is not easily observable because of the domination of the outer shell. The removal of electrons from a metal nanoparticle results in a slight red shift in the surface plasmon peak to higher wavelengths. ${ }^{49,50}$ It has been observed experimentally that the surface plasmon peak of the gold core in the Au@Ag particle is blue shifted with increasing Ag shell thicknesses until the two resonances merge into a single peak close to the resonance wavelength of a solid particle of Ag. Correspondingly, the resonance of the $\mathrm{Ag}$ shell is shifted slightly red. ${ }^{47,31-53} \mathrm{~A}$ similar behavior is observed for Ag@Au core-shell particles. We believe that these observed shifts in the resonance spectra are at least partially because of contact charging. A similar approach has been proposed earlier by Nayak and Ghosh, ${ }^{54}$ who considered the diffuse electron density profile at the boundary between the two metals instead of a sharp boundary assumed by the Mie theory. The experimentally observed blue shift of the Au core in $\mathrm{Au} @ \mathrm{Ag}$ nanorods is markedly more clear, ${ }^{47,55,56}$ most likely because of the increased contact area between the different metals, increasing the number of transferred electrons. ${ }^{18}$

In Au@Ag particles, the Fermi level of the Au core is the same as the Fermi level of the Ag shell. The question is, how does the number of electrons transferred to the gold vary with the thickness of the shell layer or the radius of the core? The previous treatment would suggest that even a monolayer of silver on gold would make the Fermi level of gold to align with the Fermi level of bulk silver, and hence the work function of a metal is not so sensitive to the three-dimensional structure of the solid. ${ }^{57}$ Of course, the silver layer has to be thick enough so that it behaves as bulk silver, but it seems that only a few monolayers are enough to recover the bulk work function. ${ }^{57}$ For example, recent computational studies have shown that a subsurface layer of a metal $\mathrm{M}$ inserted into a $\mathrm{Pd}$ particle can significantly change the work function of the $\operatorname{Pd}($ shell $)$ / $\mathrm{M}$ (monolayer)/Pd(core) particle, but the work function of bulk $\mathrm{Pd}$ is recovered by increasing the thickness of the outer Pd shell. ${ }^{58}$ In addition, the deformation of the metal structures owing to the mismatch of unit cell parameters between two different metals induces surface stress. ${ }^{3,59,60}$ This changes the chemical potential of electrons in the surface layers, ${ }^{3,59,80}$ but if the layers are thick enough, the deviation from bulk values are small.

\section{CONCLUSIONS}

Fermi level equilibration takes place upon contact of two different electric conductors (either metals or semiconductors) through electron transfer from the material with the lower work function to the other material. Most, but not all, of this transferred charge is retained at the interface as an interfacial dipole. However, the charge at the outer surfaces induces a potential on both metals, where the potential difference is equal to the work function difference in the two materials. The position of the Fermi level (and the distribution of this electrostatic potential) is strongly dependent on the surface coverage of different metals and metal facets on the structure (for spheres and surfaces this dependency is linear). Basically, the Fermi level of the structure is the weighted average work function of all the surfaces. In addition, the amount of the transferred charge depends strongly on the geometry of the system. Shifts in the Fermi level can have a drastic effect on, for example, electrocatalytic, catalytic, and optical properties of multimetallic systems; for example, shifts observed in surface plasmon resonance of core-shell nanoparticles seem to result from contact electrification. Understanding and taking advantage of the contact electrification on the material design is expected to lead to improved performance of, for example, bifunctional electrocatalysts.

In the case of two spheres of different metals, the position of the Fermi level cannot be expressed simply as the surface areaweighted average of the Fermi levels of pure metals but instead the electrostatics of the system have to be considered more carefully. Electrostatic calculations of the force between the two spheres show that the spheres will attract each other. If the separation is less than the distance of electron tunneling, the potential difference between the spheres is constant, whereas the charge depends on the separation distance. Outside of this cutoff distance, the charge remains constant, whereas the potential difference changes.

When a bimetallic particle is placed into an electrolyte solution, an electric double layer forms around it. The behavior of this double layer around $\mathrm{Ag}-\mathrm{Au}$ Janus particles of different compositions was studied by numerically solving the modified Poisson-Boltzmann equations using finite element simulations. The results show that the electric field induced by the contact electrification disappears beyond the Debye length.

\section{ASSOCIATED CONTENT}

\section{S Supporting Information}

The Supporting Information is available free of charge on the ACS Publications website at DOI: 10.1021/acs.langmuir.6b01282.

Basic concepts of the Fermi level and work function of a metal, and detailed information about the finite element simulations, including the validation of the results using the analytical solution of Laplace's equation (PDF)

\section{AUTHOR INFORMATION}

\section{Corresponding Author}

*E-mail: pekka.peljo@epfl.ch.

\section{Author Contributions}

The manuscript was written through contributions of P.P., J.A.M., and H.H.G. All authors have approved the final version of the manuscript.

\section{Notes}

The authors declare no competing financial interest.

\section{ACKNOWLEDGMENTS}

We would like to acknowledge financial support from the Swiss National Science Foundation (Grants Ambizione Energy 160553 and Solar Fuels 2000-20_152 557/1), Fondazione Oronzio e Niccolò De Nora, and EPFL. J.A.M. thanks support from the Ministry of Economy and Competitiveness and FEDER through project MAT2015-65011-P, as well as the help 
provided by Prof. B. Gimeno with the analytical solution of Laplace's equation.

\section{ABBREVIATIONS}

DFT, density functional theory; IE, ionization energy; IUPAC, International Union of Pure and Applied Chemistry

\section{REFERENCES}

(1) Stamenkovic, V. R.; Fowler, B.; Mun, B. S.; Wang, G.; Ross, P. N.; Lucas, C. A.; Marković, N. M. Improved Oxygen Reduction Activity on $\mathrm{Pt}_{3} \mathrm{Ni}(111)$ via Increased Surface Site Availability. Science 2007, 315, 493-497.

(2) Huang, X.; Zhao, Z.; Cao, L.; Chen, Y.; Zhu, E.; Lin, Z.; Li, M.; Yan, A.; Zettl, A.; Wang, Y. M.; Duan, X.; Mueller, T.; Huang, Y. HighPerformance Transition Metal-Doped $\mathrm{Pt}_{3} \mathrm{Ni}$ Octahedra for Oxygen Reduction Reaction. Science 2015, 348, 1230-1234.

(3) Jia, Q.; Liang, W.; Bates, M. K.; Mani, P.; Lee, W.; Mukerjee, S. Activity Descriptor Identification for Oxygen Reduction on PlatinumBased Bimetallic Nanoparticles: In Situ Observation of the Linear Composition-Strain-Activity Relationship. ACS Nano 2015, 9, 387400.

(4) Ferrando, R.; Jellinek, J.; Johnston, R. L. Nanoalloys: From Theory to Applications of Alloy Clusters and Nanoparticles. Chem. Rev. 2008, 108, 845-910.

(5) Chen, P.-C.; Liu, G.; Zhou, Y.; Brown, K. A.; Chernyak, N.; Hedrick, J. L.; He, S.; Xie, Z.; Lin, Q.-Y.; Dravid, V. P.; O'NeillSlawecki, S. A.; Mirkin, C. A. Tip-Directed Synthesis of Multimetallic Nanoparticles. J. Am. Chem. Soc. 2015, 137, 9167-9173.

(6) Tao, F. F. Synthesis, Catalysis, Surface Chemistry and Structure of Bimetallic Nanocatalysts. Chem. Soc. Rev. 2012, 41, 7977-7979.

(7) Sankar, M.; Dimitratos, N.; Miedziak, P. J.; Wells, P. P.; Kiely, C. J.; Hutchings, G. J. Designing Bimetallic Catalysts for a Green and Sustainable Future. Chem. Soc. Rev. 2012, 41, 8099-8139.

(8) Hakim, S. H.; Sener, C.; Alba-Rubio, A. C.; Gostanian, T. M.; O’Neill, B. J.; Ribeiro, F. H.; Miller, J. T.; Dumesic, J. A. Synthesis of Supported Bimetallic Nanoparticles with Controlled Size and Composition Distributions for Active Site Elucidation. J. Catal. 2015, 328, 75-90.

(9) Kuisma, M.; Sakko, A.; Rossi, T. P.; Larsen, A. H.; Enkovaara, J.; Lehtovaara, L.; Rantala, T. T. Localized Surface Plasmon Resonance in Silver Nanoparticles: Atomistic First-Principles Time-Dependent Density-Functional Theory Calculations. Phys. Rev. B 2015, 91, 115431.

(10) Scanlon, M. D.; Peljo, P.; Méndez, M. A.; Smirnov, E.; Girault, H. H. Charging and Discharging at the Nanoscale: Fermi Level Equilibration of Metallic Nanoparticles. Chem. Sci. 2015, 6, 27052720.

(11) Hong, S. Controversy over Voltaic Contact Phenomena, 18621900. Arch. Hist. Exact Sci. 1994, 47, 233-289.

(12) Kelvin, L. V. Contact Electricity of Metals. Philos. Mag. Ser. 5 1898, 46, 82-120.

(13) Zisman, W. A. A. New Method of Measuring Contact Potential Differences in Metals. Rev. Sci. Instrum. 1932, 3, 367-370.

(14) Melitz, W.; Shen, J.; Kummel, A. C.; Lee, S. Kelvin Probe Force Microscopy and its Application. Surf. Sci. Rep. 2011, 66, 1-27.

(15) Lacks, D. J.; Sankaran, R. M. Contact Electrification of Insulating Materials. J. Phys. D: Appl. Phys. 2011, 44, 453001.

(16) Harper, W. R. The Volta Effect as a Cause of Static Electrification. Proc. R. Soc. A 1951, 205, 83-103.

(17) Lekner, J. Electrostatics of Two Charged Conducting Spheres. Proc. R. Soc. A 2012, 468, 2829-2848.

(18) Holmberg, N.; Laasonen, K.; Peljo, P. Charge Distribution and Fermi Level in Bimetallic Nanoparticles. Phys. Chem. Chem. Phys. 2016, 18, 2924-2931.

(19) Trasatti, S.; Parsons, R. Interphases in Systems of Conducting Phases. J. Electroanal. Chem. Interfacial Electrochem. 1986, 205, 359376.
(20) Girault, H. H. Analytical and Physical Electrochemistry; EPFL Press: Lausanne, 2004.

(21) Hölzl, J.; Schulte, F. K. Work Function of Metals. In Solid Surface Physics; Hölzl, J., Schulte, F. K., Wagner, H., Eds.; Springer: Berlin, 1979; pp 1-150.

(22) Trasatti, S. The Absolute Electrode Potential: An Explanatory Note. Pure Appl. Chem. 1986, 58, 955-966.

(23) Parsons, R. The Single Electrode Potential: its Significance and Calculation. In Standard Potentials in Aqueous Solution; Bard, A. J., Parsons, R., Jordan, J., Eds.; Marcel Dekker: New York, 1985; pp 1337.

(24) Manzanares, J. A.; Reiss, H.; Heeger, A. J. Polymer LightEmitting Electrochemical Cells: A Theoretical Study of Junction Formation under Steady-State Conditions. J. Phys. Chem. B 1998, 102, $4327-4336$

(25) Sommerfeld, A. Electrodynamics; Academic Press: New York, 1964.

(26) Fall, C. J.; Binggeli, N.; Baldereschi, A. Work Functions and Surface Charges at Metallic Facet Edges. Phys. Rev. B 2002, 66, 075405 .

(27) Fall, C. Ab Initio Study of the Work Functions of Elemental Metal Crystals, Ph.D. Thesis, EPFL, 1999.

(28) Zhang, K.; Zhao, J.; Xu, H.; Li, Y.; Ji, J.; Liu, B. Multifunctional Paper Strip Based on Self-Assembled Interfacial Plasmonic Nanoparticle Arrays for Sensitive SERS Detection. ACS Appl. Mater. Interfaces 2015, 7, 16767-16774.

(29) Zhang, K.; Ji, J.; Li, Y.; Liu, B. Interfacial Self-Assembled Functional Nanoparticle Array: A Facile Surface-Enhanced Raman Scattering Sensor for Specific Detection of Trace Analytes. Anal. Chem. 2014, 86, 6660-6665.

(30) Chaudhuri, R. G.; Paria, S. Core/shell Nanoparticles: Classes, Properties, Synthesis Mechanisms, Characterization, and Applications. Chem. Rev. 2012, 112, 2373-2433.

(31) Llano, J.; Eriksson, L. A. First Principles Electrochemistry: Electrons and Protons Reacting as Independent Ions. J. Chem. Phys. 2002, 117, 10193.

(32) Taylor, J. B.; Langmuir, I. The Evaporation of Atoms, Ions and Electrons from Caesium Films on Tungsten. Phys. Rev. 1933, 44, 423458.

(33) Verhoef, R. W.; Asscher, M. The Work Function of Adsorbed Alkalis on Metals Revisited: A Coverage-Dependent Polarizability Approach. Surf. Sci. 1997, 391, 11-18.

(34) Chou, S. H.; Voss, J.; Bargatin, I.; Vojvodic, A.; Howe, R. T.; Abild-Pedersen, F. An Orbital-Overlap Model for Minimal Work Functions of Cesiated Metal Surfaces. J. Phys.: Condens. Matter 2012, $24,445007$.

(35) Sahni, V.; Perdew, J. P.; Gruenebaum, J. Variational Calculations of Low-Index Crystal Face-Dependent Surface Energies and Work Functions of Simple Metals. Phys. Rev. B 1981, 23, 6512-6523.

(36) Herring, C.; Nichols, M. H. Thermionic Emission. Rev. Mod. Phys. 1949, 21, 185-270.

(37) Rothschild, J. A.; Eizenberg, M. Work Function Calculation of Solid Solution Alloys Using the Image Force Model. Phys. Rev. B 2010, $81,224201$.

(38) Cervera, J.; Ramírez, P.; Manzanares, J. A.; Mafé, S. Incorporating Ionic Size in the Transport Equations for Charged Nanopores. Microfluid. Nanofluid. 2009, 9, 41-53.

(39) Cervera, J.; García-Morales, V.; Pellicer, J. Ion Size Effects on the Electrokinetic Flow in Nanoporous Membranes Caused by Concentration Gradients. J. Phys. Chem. B 2003, 107, 8300-8309.

(40) Cervera, J. Ion Size Effects on the Current Efficiency of Narrow Charged Pores. J. Membr. Sci. 2001, 191, 179-187.

(41) Cervera, J.; Manzanares, J. A.; Mafé, S. Ion Size Effects on the Streaming Potential of Narrow Charged Pores. Phys. Chem. Chem. Phys. 2001, 3, 2493-2496.

(42) Bazant, M. Z.; Kilic, M. S.; Storey, B. D.; Ajdari, A. Towards an Understanding of Induced-Charge Electrokinetics at Large Applied Voltages in Concentrated Solutions. Adv. Colloid Interface Sci. 2009, $152,48-88$. 
(43) Nightingale, E. R. Phenomenological Theory of Ion Solvation. Effective Radii of Hydrated Ions. J. Phys. Chem. 1959, 63, 1381-1387.

(44) Aguilella-Arzo, M.; Andrio, A.; Aguilella, V. M.; Alcaraz, A. Dielectric Saturation of Water in a Membrane Protein Channel. Phys. Chem. Chem. Phys. 2009, 11, 358-365.

(45) Lebedev, K.; Mafé, S.; Alcaraz, A.; Ramírez, P. Effects of Water Dielectric Saturation on the Space-Charge Junction of a Fixed-Charge Bipolar Membrane. Chem. Phys. Lett. 2000, 326, 87-92.

(46) Varghese, J.; Wang, H.; Pilon, L. Simulating Electric Double Layer Capacitance of Mesoporous Electrodes with Cylindrical Pores. J. Electrochem. Soc. 2011, 158, A1106.

(47) Cortie, M. B.; McDonagh, A. M. Synthesis and Optical Properties of Hybrid and Alloy Plasmonic Nanoparticles. Chem. Rev. 2011, 111, 3713-3735.

(48) Rodríguez-González, B.; Burrows, A.; Watanabe, M.; Kiely, C. J.; Liz Marzán, L. M. Multishell Bimetallic AuAg Nanoparticles: Synthesis, Structure and Optical Properties. J. Mater. Chem. 2005, $15,1755-1759$.

(49) Templeton, A. C.; Pietron, J. J.; Murray, R. W.; Mulvaney, P. Solvent Refractive Index and Core Charge Influences on the Surface Plasmon Absorbance of Alkanethiolate Monolayer-Protected Gold Clusters. J. Phys. Chem. B 2000, 104, 564-570.

(50) Mulvaney, P.; Pérez-Juste, J.; Giersig, M.; Liz-Marzán, L. M.; Pecharromán, C. Drastic Surface Plasmon Mode Shifts in Gold Nanorods Due to Electron Charging. Plasmonics 2006, 1, 61-66.

(51) Wang, X.; Zhang, Z.; Hartland, G. V. Electronic Dephasing in Bimetallic Gold-Silver Nanoparticles Examined by Single Particle Spectroscopy. J. Phys. Chem. B 2005, 109, 20324-20330.

(52) Morriss, R. H.; Collins, L. F. Optical Properties of Multilayer Colloids. J. Chem. Phys. 1964, 41, 3357-3362.

(53) Gonzalez, C. M.; Liu, Y.; Scaiano, J. C. Photochemical Strategies for the Facile Synthesis of Gold-Silver Alloy and Core-Shell Bimetallic Nanoparticles. J. Phys. Chem. C 2009, 113, 11861-11867.

(54) Nayak, M. K.; Ghosh, S. K. Optical Properties of Bimetallic Nanospheres: Effect of Diffuse Electron Density Profiles at the Boundary Surfaces. J. Chem. Phys. 2009, 130, 204702.

(55) Xiang, Y.; Wu, X.; Liu, D.; Li, Z.; Chu, W.; Feng, L.; Zhang, K.; Zhou, W.; Xie, S. Gold Nanorod-Seeded Growth of Silver Nanostructures: From Homogeneous Coating to Anisotropic Coating. Langmuir 2008, 24, 3465-3470.

(56) Becker, J.; Zins, I.; Jakab, A.; Khalavka, Y.; Schubert, O.; Sönnichsen, C. Plasmonic Focusing Reduces Ensemble Linewidth of Silver-Coated Gold Nanorods. Nano Lett. 2008, 8, 1719-1723.

(57) Davidson, E. R. Alloy Work Functions: Extended Hückel Calculations for $\mathrm{Ag}-\mathrm{Au}$ and $\mathrm{Cu}-\mathrm{Au}$ Clusters. J. Vac. Sci. Technol. 1976, 13, 209-213.

(58) Ding, Z.-B.; Wu, F.; Wang, Y.-C.; Jiang, H. Theoretical Studies of the Work Functions of Pd-Based Bimetallic Surfaces. J. Chem. Phys. 2015, 142, 214706.

(59) Jia, Q.; Segre, C. U.; Ramaker, D.; Caldwell, K.; Trahan, M.; Mukerjee, S. Structure-property-activity Correlations of Pt-Bimetallic Nanoparticles: A Theoretical Study. Electrochim. Acta 2013, 88, 604-613.

(60) Kitchin, J. R.; Nørskov, J. K.; Barteau, M. A.; Chen, J. G. Role of Strain and Ligand Effects in the Modification of the Electronic and Chemical Properties of Bimetallic Surfaces. Phys. Rev. Lett. 2004, 93, 156801 . 